\title{
Determination of the hyperelastic material behavior of hydrogel specimens for expandable lens implants
}

\begin{abstract}
:
Theoretical and experimental investigations were carried out for development of human lens implants made from hydrogel material. The material properties were measured and implemented in a Finite element model. Though the material is still too stiff for accommodative lenses, the theoretical and experimental foundations for the implant development were established.
\end{abstract}

Keywords: Biomechanics, Ophthalmology, Material testing

https://doi.org/10.1515/cdbme-2019-0088

\section{Introduction}

The development of accommodative intraocular lenses has not achieved a sustainable success so far, though numerous concepts were developed and tested. Especially the replacement of the lens material, which is becoming harder with the age, remains still a competitive concept due to its simplicity.

The filling of the lens capsule with curing polymer was further enhanced, however, the risk of the exact dosing of the lens content and the requirement of capsular sealing remain as well as the uncertainty of ensuring the optical quality of the lens. Therefore, an alternative concept of lens implants made of expandable biocompatible hydrogels was investigated, which allows a relatively simple implantation and an exactly defined lens shape.

\footnotetext{
*Corresponding author: Heiner Martin: University Medical Center Rostock, Institute for Biomedical Engineering, F.Barnewitz-Str. 4, Rostock, Germany, e-mail: heiner.martin@unirostock.de

Olga Sahmel, Thomas Eickner, Niels Grabow: University Medical Center Rostock, Institute for Biomedical Engineering, Rostock, Germany

Christine Kreiner: KreCo $\mathrm{GmbH}$, Hennigsdorf, Germany

Rudolf Guthoff: Department of Ophthalmology, University

Medical Center Rostock, Germany
}

Finite element investigations of lens sections as well as material compression tests between parallel plates are used to check the material data of a given lens material and its usability for artificial intraocular lenses made of hydrogel.

\section{Material and Methods}

\subsection{Determination of material data by compression test}

An important point in this concept is to ensure the optical and mechanical material properties, which allow a deformation of the lens with accommodation. From earlier finite element investigations (e.g. Burd et al [1]) and comparison with measurements the required material properties of the human native lens and its capsule are approximately known.

Hence, the hyperelastic material law of hydrogel test specimens was determined for lens implants in compression tests and evaluated in finite element models of hydrogel test specimens, as well as of lenses.

Cylindrical test specimens with diameters of $10 \mathrm{~mm}$ and $2 \mathrm{~mm}$ height (in unexpanded condition) were tested in compression tests. Specimens of the hydrogel were tested in pressure test after an expansion time of at least $24 \mathrm{~h}$ between two parallel plates in a material testing machine. The nominal stress-strain-diagrams show a hyperelastic material behaviour (Figure 1).

In order to evaluate the influence of the transverse contraction restraint due to friction on the results, finite element models of the pressure tests were analysed with and without transverse contraction.

\subsection{Investigation of deformation of lens implant specimens in finite element models}

From the shape change of a lens implant due to the centrifugal load in a specially designed test device such as 
that by Burd [1] the accommodation ability of lenses can be determined. The lens implant is rotated with revolution speeds of about $1000 \mathrm{~min}^{-1}$ around the optical axis and the lens contour is photographically recorded to determine the shape change, the axial lens thickness and the lens diameter. For an exact comparison of the uniaxial pressure test data, a finite element model of the lens in the test device under centrifugal load is used for comparison with measured results. The lens implant is analysed under gravity load, as well as under centrifugal load. For the shape of the lens, the data determined for a 29 year old lens according to Burd's model [2] were used.

\section{Results and Discussion}

The material data determined by pressure tests in the material testing machine show that the given hydrogel is still too stiff to use it for lens polymer. The values of the secant modulus are with about $20 \mathrm{kPa}$ considerably higher than the values for

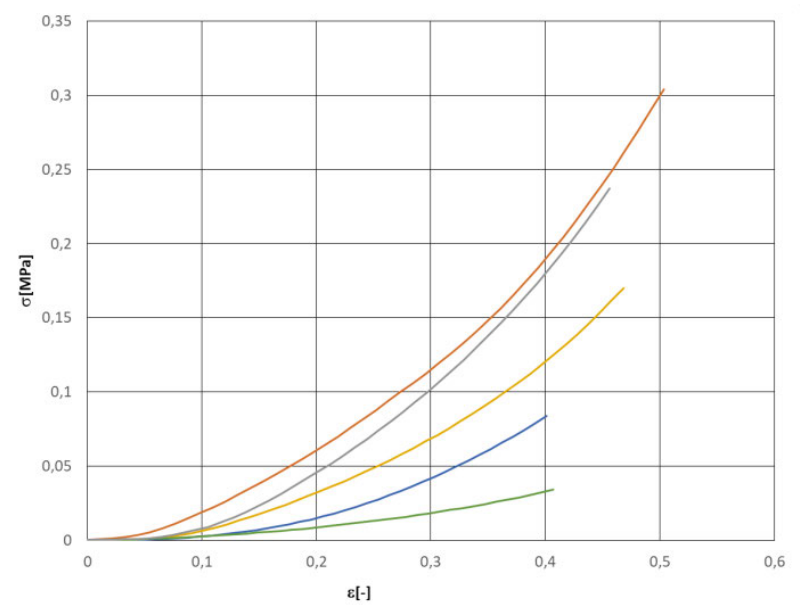

Figure 1: Nominal stress-strain diagram for hydrogel specimens of various charges (These material charges differ in their water uptake and in their composition; they represent the typical range of material properties)

nucleus $(0.55 \mathrm{kPa})$ and cortex $(3.42 \mathrm{kPa})$ for a 29 year old lens. A considerably softer hydrogel material has to be developed, taking also the optical refraction index of the natural lens material into consideration.

Nevertheless, a two dimensional axisymmetric finite element model for the investigation of lens implants under centrifugal load was developed, which can be used for the investigation of implants under centrifugal load.
The model has the shape of the lens according to Burd, however, it can be adapted to the contours of the lens implant. A comparison between measurements and computed results of the deformation with radial load on the lens implant is thereby possible (Figure 2).
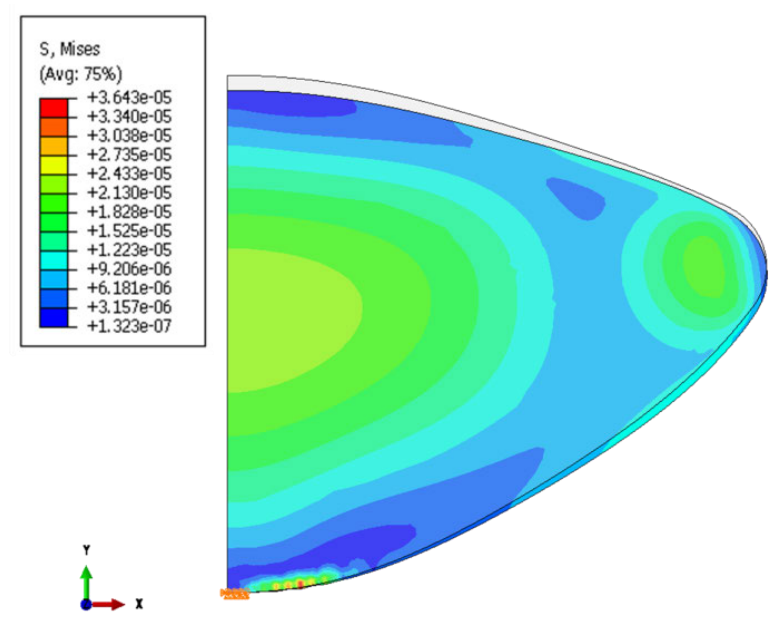

Figure 2: Computed v. Mises stress distribution $\left[\mathrm{N} / \mathrm{mm}^{2}\right]$ in the model of the lens in the test device under radial centrifugal load

\section{Author Statement}

Research funding: The investigations presented were supported by the Bundesministerium für Bildung und Forschung (BMBF, German Federal Ministry of Research and Technology) within RESPONSE joint project (FKZ: 03ZZ0912A). Conflict of interest: Authors state no conflict of interest. Informed consent: Informed consent has been obtained from all individuals included in this study. Ethical approval: The research related to human use complies with all the relevant national regulations, institutional policies and was performed in accordance with the tenets of the Helsinki Declaration, and has been approved by the authors' institutional review board or equivalent committee.

\section{References}

[1] Burd HJ, Wilde GS, Judge SJ. An improved spinning lens test to determine the stiffness of the human lens. Exp Eye Res. 92(2011)1, 28-39.

[2] Burd HJ, Judge SJ, Cross JA: Numerical modelling of the accommodating lens. Vision Research, 42 (2002) 18, 22352251. 